\title{
Role of classification in grinding using the electromagnetic mill. A case study
}

\author{
Marta Wolosiewicz-Glab ${ }^{1, a}$, Dariusz Foszcz ${ }^{1}$, Tomasz Gawenda ${ }^{1}$, and Szymon Ogonowski² \\ ${ }^{1}$ AGH University of Science and Technology, Faculty of Mining and Geoengineering, Department of Environmental \\ Engineering and Mineral Processing, Poland \\ ${ }^{2}$ Silesian University of Technology, Faculty of Automatic Control, Electronics and Computer Science, Institute of \\ Automatic Control, Poland
}

\begin{abstract}
In the process of classification, one of the methods used to evaluate the effectiveness classifiers is to plot a separation curve, which determines the size of seprated particles and characterizes the process accuracy. The article presents an assessment of the impact of parameters on grinding and classification using an electromagnetic mill. The results allow a detailed assessment of the effectiveness and efficiency of the device and facilitate the optimization of the grinding process by establishing an appropriate control algorithms.
\end{abstract}

\section{Introduction}

The quality of the products obtained from the preparation processes depends primarily on the correct selection of equipment and auxiliary devices depending on the type of raw material processed. Assuming a specific technological process, usually one takes into account the characteristics of individual machines performance, which depends, among other factors, on the physico-mechanical feed properties and quantities of the designable and steerable machines' parameters [1,2]. It turns out, however, that obtaining products with the appropriate parameters is also affected by the conduct of the technological process that is the number of grinding stages, technological cycles, flow control of material to the selected devices etc. [1,2]. For example, in the processes of crushing and grinding during the production of crushed aggregates closed technological cycles are often used. It is so called a grinding process in a closed circuit. There are also technological systems with open flow of material. Such systems have the advantage over closed cycles in terms of performance, but often the obtained products have lower quality $[1,2]$.

Figure 1 shows the technological cycles of grinding with screening grain classification but systems of this type can be also used for other devices, such as mill-separator [1,2].

\footnotetext{
${ }^{\text {a }}$ Corresponding author: wolosiewiczm@gmail.com
} 
a)

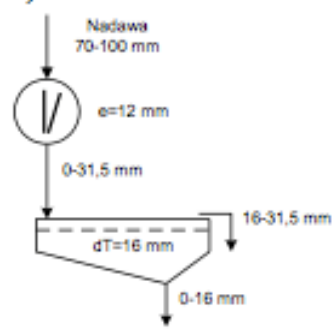

b)

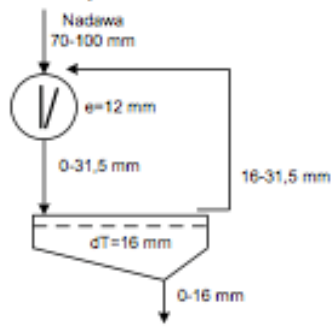

c)

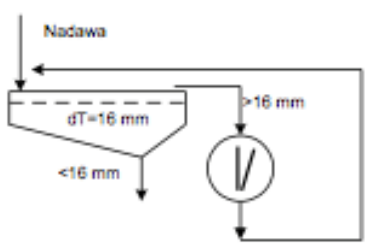

Figure 1. Technological cycles: a) open cycle, b, c) closed cycles [1, 2].

\section{Inertial-impingement separator used in electromagnetic mill}

An inertial-impingement separator with a cyclone has been designed, basing on computational flow dynamics (CFD) for explicit performance parameters and particle size of individual streams. Figure 2 shows the chosen sorted grain sizes that result from the CFD simulation of the flow.

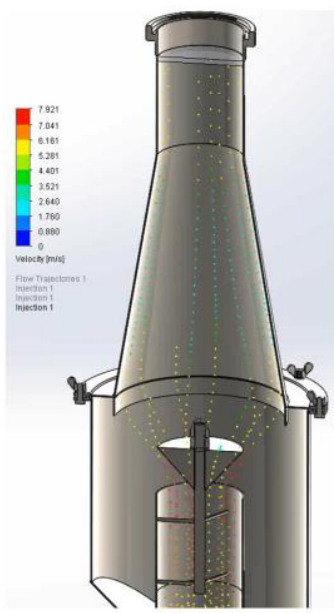

40um

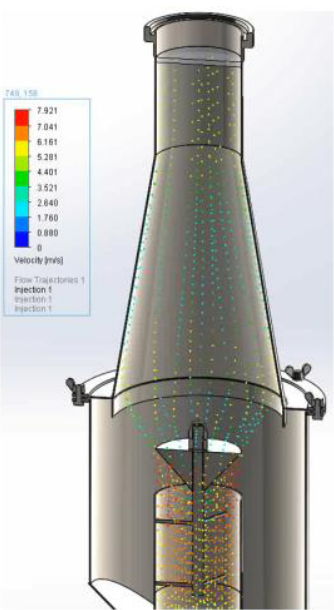

50um

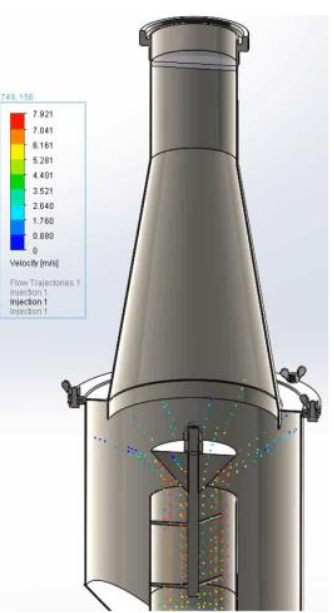

60um

Figure 2. Results of flow simulation CFD for different sizes of feed grains led to separator (courtesy of Pro-mill cyclone contractor).

The separator is designed to allow a change of the sorted grain size by changing the position (mounting height) of the impactor or replacement with another element with different opening angle. Figure 3 shows the design, fabrication and impingement component of this type of separator. As a result of shredding and other physical and chemical processes, a mixture of solid particles of different sizes and shapes is obtained. Very often a specific grain size or range of sizes are required. The classification can be done by [3]:

- in the gas stream - pneumatic classification,

- in the stream of liquid - hydraulic classification,

- $\quad$ sieve - screening.

In the system with an electromagnetic mill, the inertial-impingement separator has been designed, shown in the system functional layout in Figure 4. 
a)

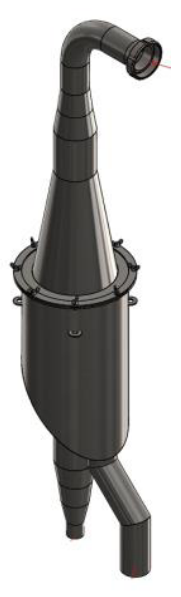

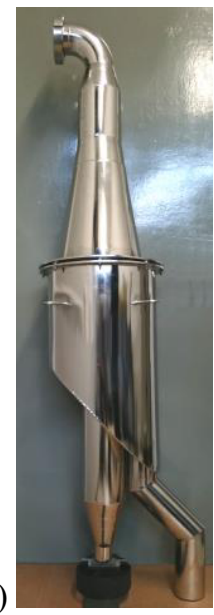

c)

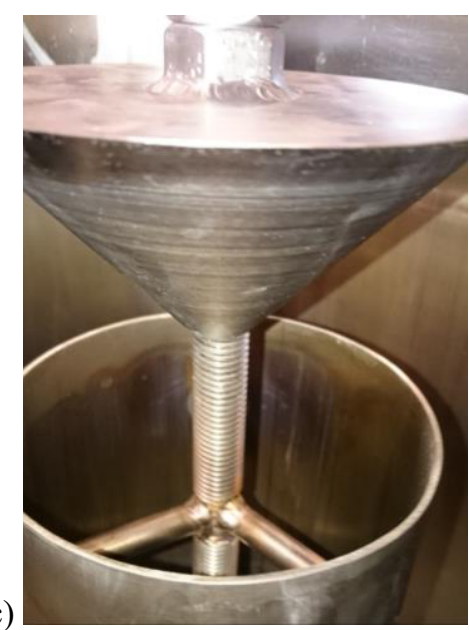

Figure 3. Inertial-impingement separator: design (a), fabrication (b), impingement element (c) (courtesy of Promill - cyclone contractor).

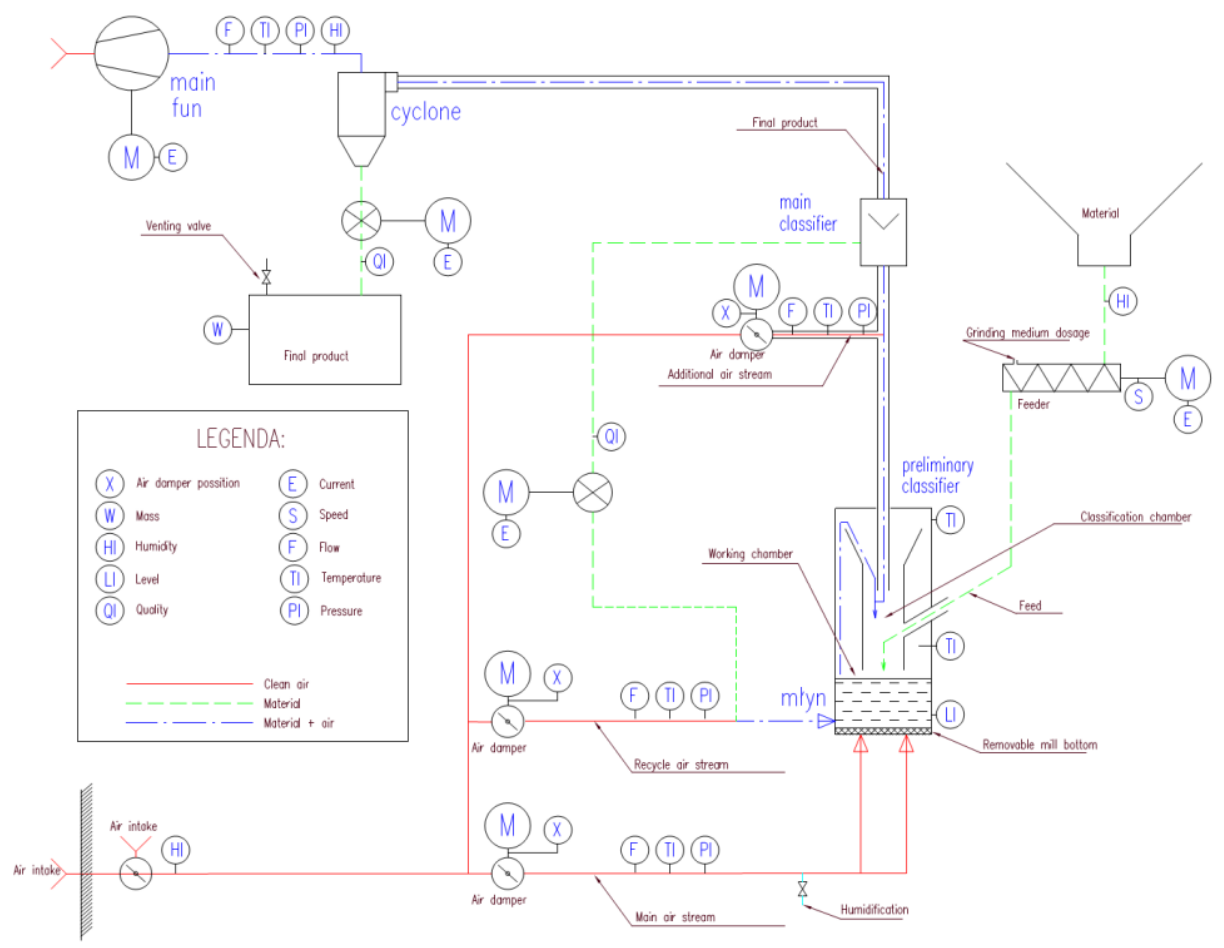

Figure 4. Functional structure of dispensing system of grinding media along with classification [4]

Inside of the electromagnetic mill system, there are classifiers: integrated internal classifier and inertial-impingement. They create a closed system. Over the initial classifier, the inertial-impingement separator was placed from which the recycle flow is led back into the mill, feeded from the bottom of the working chamber (Fig. 5). 


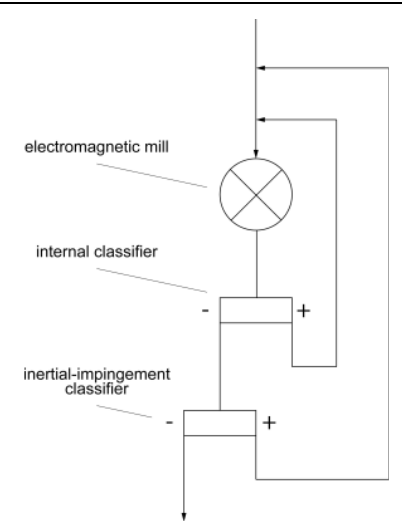

Figure 5. Functional structure of classifiers in electromagnetic mill.

A properly selected classification device should give a sharp separation effect curve, which means that the larger particles should not blend with the fine product as well as the fine particles corresponding to the final product should not blend into recycle flow coming out from the classifier $[1,2]$.

Multiplicity of the circuit is defined as the ratio of the amount of material recycled to the quantity of new feed. Circuit multiplicity reaches high values in the milling processes. In practice, for grinding in mills cement or limestone it is equal to 2-2.6. In case of the material processed in crushers, these values are much lower [5].

In the case of separation of small grains blends (approximately $<1 \mathrm{~mm}$ ) and very small $(<0.1 \mathrm{~mm})$, the classification process is often carried out in either aqueous or air medium. During the processes of grading, different forces are acting (centrifugal, resistance of the medium, Coriolis, gravity, buoyancy, gradient resulting from changes in grains concentration) on the particle [1, 2]. Differences in classifiers' design determine the values and directions of these forces as well as the quantitative and qualitative characteristics of the implemented process.

In case of an ideal material separation with sieves or classifiers, grains characterized by a distribution parameter value (e.g. size or weight) lower than a predetermined, are in one product and the other in the second. Under real conditions, the separation is never perfect, and part of the grains go to the wrong product. To characterize the accuracy of the separation effect, we introduce separation values or separation efficiency for the classes or fractions. It is defined as the ratio of mass of the end product to the weight of that class in the feed $[6,7]$ :

$$
\begin{aligned}
& T\left(r_{i}\right)=\frac{Q_{P 1} \cdot a_{i_{P 1}}}{Q_{N} \cdot a_{i_{N}}}=\gamma_{P 1} \cdot \frac{a_{i_{P 1}}}{a_{i_{N}}} \\
& \tau\left(r_{i}\right)=\frac{Q_{P 2} \cdot a_{i_{P 2}}}{Q_{N} \cdot a_{i_{N}}}=\gamma_{P 2} \cdot \frac{a_{i_{P 2}}}{a_{i_{N}}}
\end{aligned}
$$

where:

$T(r), \tau(r) \quad$ - separation curves,

i

- the average value of the characteristics under which the separation takes place,

$a_{i_{N}}$

- number of fractions $(i=1,2, \ldots, k)$,

$-\mathrm{i}$-th fraction in the feed

$a_{i_{P 1}}$

$-i$-th fraction in the first product

$a_{i_{P 2}}$

$Q_{N}$

$Q_{P 1}$

$Q_{P 2}$

$-\mathrm{i}$-th fraction in the second product

- feed weight

- first product weight

- second product weight 
$\gamma_{P 1} \quad-$ first product flow off

$\gamma_{P 2} \quad-$ second product flow off.

Each classifier has a specific separating ability, which operates at a predetermined separation boundary or otherwise, at a given sorted grain. The sorted grain quantity is the particle diameter expressed in eg. $\mu \mathrm{m}$ (micrometers), for which the probability to be found in iether lower or upper fraction is equal to $50 \%$ [6].

The separating ability of the classifier or separation effect curve is a function of the separation depending on the diameter of the grain. It is most commonly presented in the form of a curve in a cordinate system in which the abscissa indicated the grain size and the ordinate the degree of separation in [\%]. The separation effect curve is not a curve of particle size distribution, it is a set of points representing the infinitely narrow grain class with an average grain size $d$ splits into upper and lower product [6].

Probable error $\mathrm{E}_{\mathrm{p}}$, is defined as [8]:

$$
E_{p}=\frac{\rho(T=75)-\rho(T=25)}{2}
$$

where: $\rho(T=75)=\rho_{75}$ and $\rho(T=25)=\rho_{25}[9]$ and represent readings from the distribution curve of the density values for the ordinates $75 \%$ and $25 \%$.

Probable error $E_{p}$ is a threshold density, which may or may not be exceeded with the same probability by the individual grains with disperse of elementary fraction of specific density. The $E_{p}$ value is smaller, then the separation is more accurate. For perfect separation $E_{p}=0$.

Imperfection I, a benchmark for different separation density distribution, expressed as:

$$
I=\frac{E_{p}}{\rho_{r}}
$$

where $\rho_{r}=\rho_{50}$ is a reading from the distribution curve of the density value for the ordinate $50 \%$ [3].

\section{Electromagnetic mill. Separation effect curves for inertial- impingement classifier}

Figure 6 shows the separation curve for the electromagnetic mill with inertial-impingement classifier and particle size distribution of up to $5 \mathrm{~mm}$. Values obtained for the separation curve were:

- $\quad$ probable error: 0.1

- imperfection: $I=0.05$.

In an ideal material separation for the sieves or classifiers, grains having a distribution parameter value (eg. size or weight) less than a predetermined, are placed in one product and all other in the second. In terms of actual separation, it is never perfect, and part of the grains goes to the wrong product. To characterize the accuracy of separation effect, the number of separation effectiveness for classes or fractions has been introduced and presented in Fig. 6.
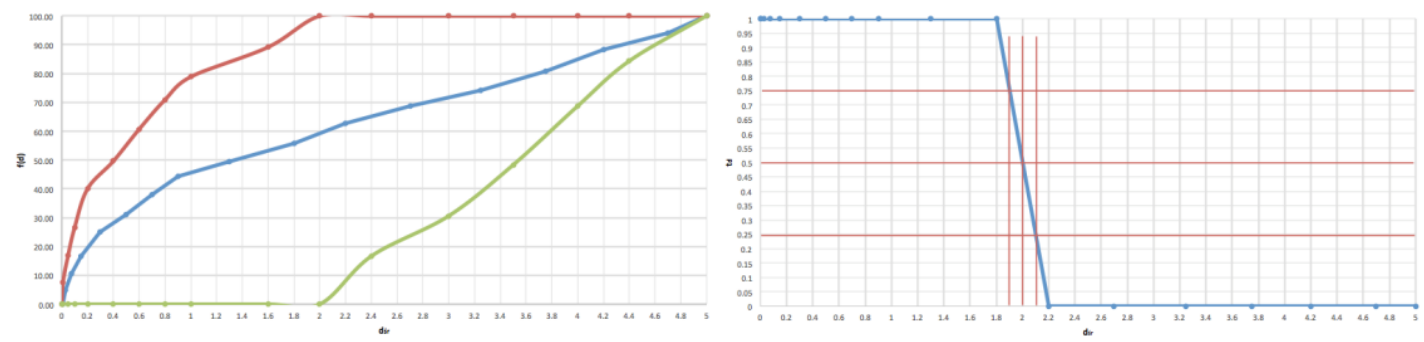

Figure 6. a) Feed, recycle, overflow curves; b) separation effect curve. 
Figure 7 shows the separation curve for the electromagnetic mill with inertial-impingement classifier and particle size distribution of up to $5 \mathrm{~mm}$. Values obtained for the separation curve were:

- $\quad$ probable error: 0.3625

- imperfection: $I=0.18125$.

From Figure 7 one can see that the imperfection, relative to the ideal system, has risen, therefore the classifier works less efficiently and there is a risk of recycle which will reduce the efficiency of the mill.
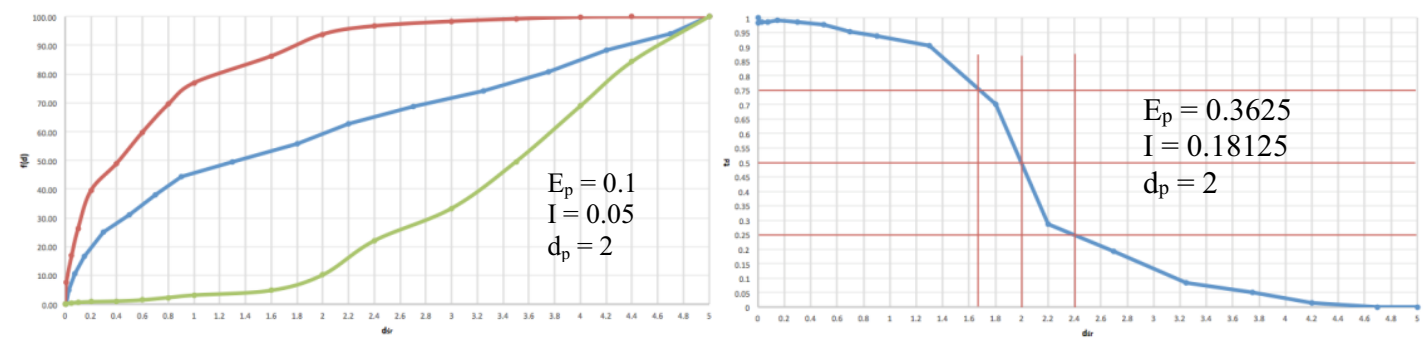

Figure 7. a) Feed, recycle, overflow curves; b) separation effect curve.

Figure 8 shows the separation curve for the electromagnetic mill with inertial-impingement classifier and particle size distribution of up to $5 \mathrm{~mm}$. Values obtained for the separation curve were:

- probable error: 0.925

- imperfection: $I=0.4625$.

From Figure 8 one can see that imperfection reaches the highest value among all presented in the paper separation effect curves, and the recycle gets a larger share in the feed than the overflow, which causes a significant reduction in efficiency of grinding system.

A significant amount of recycled material caused by the mismatched parameters of the classifier is a significant burden for the system of the mill, the separator and the transport equipment.
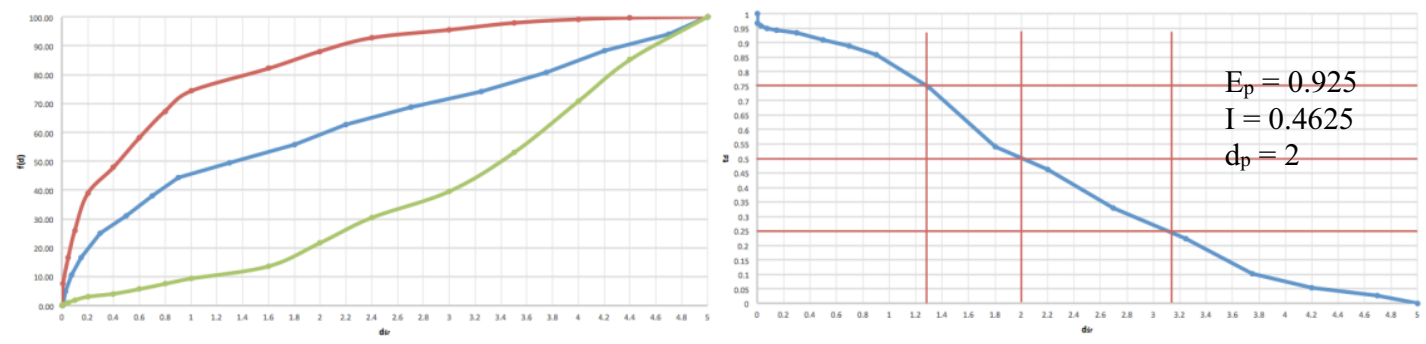

Figure 8. a) Feed, recycle, overflow curves; b) separation effect curve.

Figure 9 shows the separation curve for the electromagnetic mill with inertial-impingement classifier and particle size distribution of up to $5 \mathrm{~mm}$. Values obtained for the separation curve were:

- probable error: 0.65

- imperfection: $\mathrm{I}=0.325$.

Figure 10 depicts the content of the particles with the diameter larger than $d_{\mathrm{p}}$ in the overflow. It is easy to notice that the content of such grains decreases when the imperfection increases.

Thanks to the simulations, we can obtain the information related to the parameters of operation of the system what enabled the introduction of appropriate control in order to improve the performance of grinding and classification. Matching appropriate parameters of the classifier allows to optimize system operation. Such matching is possible and much easier due to the separation effect curve. 

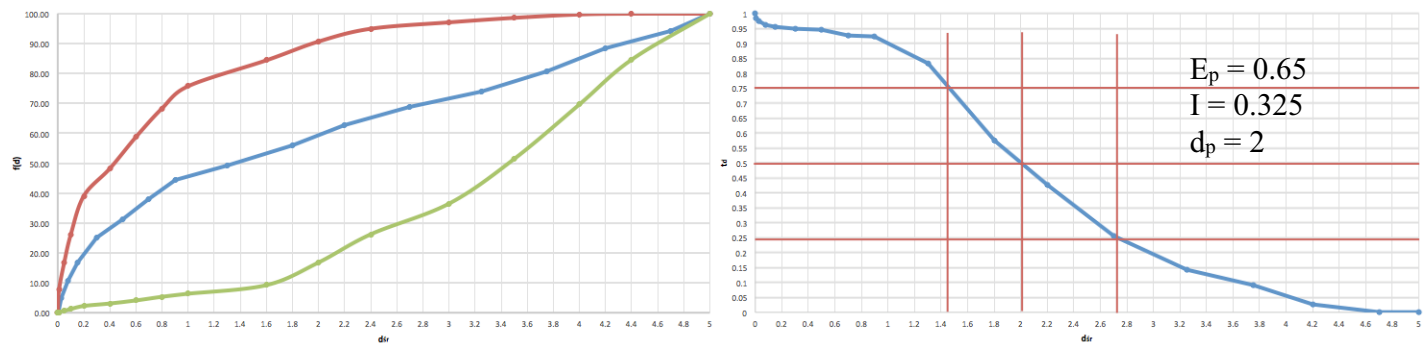

Figure 9. a) Feed, recycle, overflow curves; b) separation effect curve.

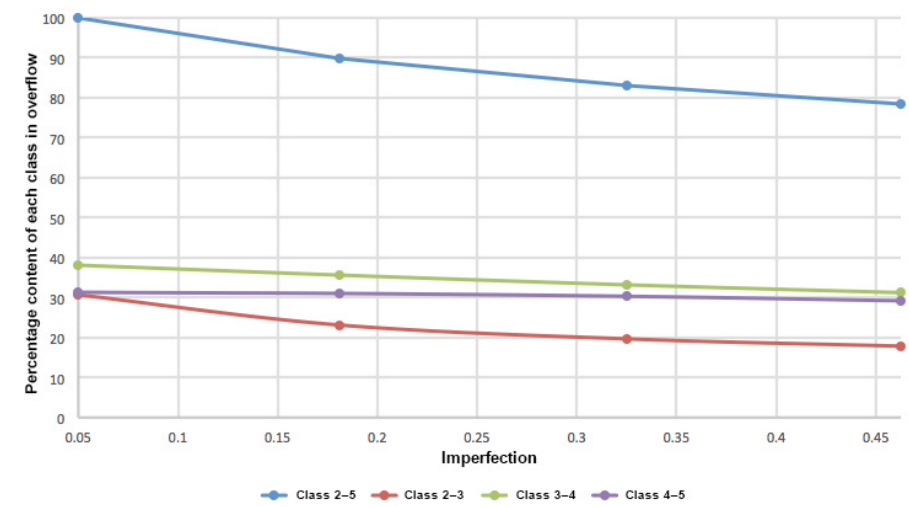

Figure 10. Content of grain classes in overflow. Only size fractions with the diameter of grain higher than $d_{p}$.

\section{Summary}

When designing a classifying system for the electromagnetic mill, the goal is to obtain the most efficient operation of the mill. With a separation effect curves, it is possible to make simulation of the grinding and classification process and determine the size of recycle flow which would allow for introduction of appropriate control algorithms that guarantee the correct operation of the mill and the entire system. With the calculations, one obtaines characteristics of the recycle value. In the review of the classification devices for fine-grained products, to predict the final products of the electromagnetic mill, one should use the separation effect curves, which provide opportunities to optimize the operation of the grinding-classification system. One can read from them the sorted grain size and get information about the accuracy of the implemented process. Made in the article comparisons of the efficiency of the device with different parameters, permit us to determine the impact of the classification efficiency on the level of recycle that will determine the efficiency of the whole grinding and classification system. The paper shows how to determine the optimum operating conditions of abruptly-inertial classifier, which was built specifically for the designed electromagnetic mill. The simulations allow to determine the functional dependencies for the actual classification process and enable the development of algorithms for control system in order to obtain the best product quality in the real system. This will be a subject of further research.

\section{Acknowledgement}

Development of the article has been funded in the framework of the project: PBS3/B3/28/2015. 


\section{References}

1. T. Gawenda, Monograph No. 304 (Wydawnictwo AGH, 2015)

2. Z. Naziemiec, T. Gawenda: in Proccedings of VII Conference Kruszywa mineralne: surowce rynek - technologie - jakosc (Oficyna Wydawnicza PWR, 2007)

3. J. Warach, Aparatura chemiczna i procesowa (Oficyna Wydawnicza Politechniki Warszawskiej, Warszawa, 1996)

4. M. Pawelczyk, Z. Ogonowski, Sz. Ogonowski, D. Foszcz, D. Saramak, T. Gawenda, Patent No. P.413041 (6.07.2015)

5. T. Gawenda, Monograph No. 304 (Wydawnictwo AGH, 2015)

6. Z. Naziemiec, D. Saramak, Min. Sci., 22(1), 119 (2015)

7. Z. Blaschke, M. Brozek, E. Mokrzycki, Z. Ociepa, T. Tumidajski, Górnictwo Cz. V. Zarys technologii procesów przeróbczych (Skrypt AGH, no 931, 1983)

8. W. Stepinski, Wzbogacanie grawitacyjne (PWN, Warszawa, 1964)

9. A. Surowiak, Ph.D. thesis, (AGH University of Science and Technology, 2006) 